\title{
Powering Wireless Sensor Networks with Solar Generated RF
}

\author{
Amar Esse Ahmed \\ Department of Electrical and Computer \\ Engineering \\ International Islamic University Malaysia \\ Kuala Lumpur, Malaysia \\ amaresseahmed@gmail.com
}

\author{
Khaizuran Abdullah \\ Department of Electrical and Computer \\ Engineering \\ International Islamic University Malaysia \\ Kuala Lumpur, Malaysia \\ khaizuran@iium.edu.my
}

\author{
Mohamed Hadi Habaebi \\ Department of Electrical and Computer \\ Engineering \\ International Islamic University Malaysia \\ Kuala Lumpur, Malaysia \\ habaebi@iium.edu.my
}

\author{
Huda Adibah Ramli \\ Department of Electrical and Computer Engineering \\ International Islamic University Malaysia \\ Kuala Lumpur, Malaysia \\ hadibahmr@iium.edu.my
}

\author{
Mohamad Huzaimy Jusoh \\ Centre for Satellite Communication Faculty of Electrical Engineering \\ Universiti Teknologi MARA \\ Shah Alam, Selangor, Malaysia \\ huzaimy@uitm.edu.my
}

\begin{abstract}
Powering Wireless sensor networks (WSN) is one of the most researched topics in the wireless field. Due to the density of such networks, conventional power sourcing using wires is not feasible. Furthermore, a sensor node (SN) in a WSN consumes small amounts of energy. Therefore, the $\mathrm{SN}$ can be charged wirelessly using a radio frequency (RF) power transmitter. This paper presents a solar-powered simultaneous wireless information and power transfer (SWIPT) transmitter design with dynamic power allocation (DPA). Moreover, a receiver design with separate energy harvesting (EH) and information decoding (ID) circuitries is adopted. DPA carries the communication signal on low-power subcarriers around the carrier frequency, while the energy signal is carried on a highpower continuous wave $(\mathrm{CW})$ centered at the carrier frequency. DPA reduces the interference to external networks by limiting the high power signal to a few subcarriers. Furthermore, the split receiver design increases the harvested power at the EH circuit reservoir.
\end{abstract}

Keywords-Dynamic Power Allocation, Solar energy, RF power transfer. PV cell.

\section{INTRODUCTION}

The recent developments in the internet of things (IoT) field led to the spread of low-power wireless sensor networks (WSN) [1]. The biggest hurdle against any wide-scale implementation of WSNs is power sourcing [2]. Furthermore, a sensor node $(\mathrm{SN})$ can only contain a small battery due to space and budget constraints. When the SN battery is depleted, the battery replacing cost may exceed the cost of the SN itself. Moreover, it is not economically feasible to extend a physical wire to hundreds or even thousands of SNs [3]. Therefore, the only solution to maintain the $\mathrm{SNs}$ for extended periods is through charging them wirelessly [4].

One way to charge these SNs is to charge them using radiofrequency (RF). In other words, an Access point (AP) can be used to send energy and information to the SN. Moreover, due to the well-established inverse square law, most of the RF power is lost in the transmission medium requiring the AP to transmit at relatively higher power levels compared to the power used for information communication. Accordingly, connecting the RF transmitter to a sustainable energy source is necessary to realize green WSNs. Different sustainable energy sources can be harvested, such as solar and mechanical energy.

Mechanical energy is generated from the natural flow of water, air, and vibration from moving objects such as cars moving across a bridge. Furthermore, mechanical energy sources differ in their effectiveness from most effective and reliable, e.g., hydropower, to the least effective, for instance, vibration. Vibration is converted into electricity through a piezoelectric material that generates electrical current when pressure is applied. Mechanical energy sources have moving parts making it difficult to integrate them in WSNs. Accordingly, solar energy is the most suitable candidate to support an RF-powered energy harvesting (EH) WSN due to the solar cell compactness [4].

According to its behavior, harvestable energy sources can be generally categorized into the following:

1: Unpredictable and uncontrollable: In this category, the energy source has no known pattern. It's practically completely random. Therefore, it cannot be controlled, making energy on demand out of the topic. An example of this category is a piezoelectric generator [5].

2: Predictable and uncontrollable: The energy can be predicated using simple mathematical models with sufficient accuracy. However, this kind of energy cannot be monitored or generated on demand - for example, solar energy [6].

3: controllable and predictable: Energy production is predictable and controllable. In this category, the energy source is controlled by the network and delivered based on demand. An example of this kind of energy is a wireless energy transmitter [7].

The rest of the article is organized as follows: section two presents a brief overview of solar energy, section three discusses RF energy, section four presents solar integration into RF-powered WSN, section five illustrates the proposed transmitter design. Finally, section six concludes the paper.

\section{SOLAR ENERGY}

One of most widely harvested radian energy sources is light energy. The radiant light source can be natural, like solar or artificial, for example, a light bulb. Solar energy is mainly harvested through concentrated solar power (CSP) plants and Photovoltaic (PV) solar panels. The CSP involves an array of reflecting mirrors that concentrates the solar light into a receiver, which converts the concentrated solar light into heat to run a steam turbine. Thus, due to the complexity of the CPS its highly unlikely to be considered for energy harvesting WSNs. In PV solar panels, solar radiation is converted to electricity through a semiconductor device called a PV cell. 
The cell's electrical output depends on factors including the insolation intensity and ambient temperature [8]. Moreover, a PV cell size can vary widely depending on the application. These applications vary from powering thousands of households and satellites to powering small devices such as calculators and wristwatches.

Solar power is considered a promising inexhaustible green energy source, as it involves no pollution in the energy production process [9]. In addition, PV solar transducers involve no moving parts, which helps prolong its life span, reduce its maintenance cost and increase its reliability. Solar radiation is converted to electricity using a basic solid-state device. Furthermore, Solar power is decentralized, implying that the solar cell can be implemented in rural areas [10] where the power grid is not available. Similarly, a solar cell can be deployed directly on the energy-consuming device, for example, a solar-powered lamppost. Accordingly, the wiring problem of a large number of lampposts and similar devices is solved. However, solar energy has limited temporal availability, as the sun is only visible during the daytime [11]. Hence energy storage systems are essential for the round-theclock availability of solar-generated electricity [12].

The PV panels are one of the most large-scale deployed renewable technologies. Furthermore, the number of electronic devices that use solar power is ever increasing due to their scalability as the energy production of the PV panel depends heavily on the PV size, thus if you have a small device that consumes low energy, then you can install a small PV panel on it. Moreover, the efficiency of the energy conversion of the cell depends on the type of material used to produce the panels. For example, thin-film cells have a conversion efficiency of 8 to $13.2 \%$, while mono cells have a conversion efficiency of $15-24 \%$ [12].

PV cells are deployed outside where direct sunlight with high intensity is available. Sometimes the PV cells can be deployed in an indoor environment where the artificial light intensity is relatively high such as in industrial plants. The typical outdoor solar power intensity is $500 \mathrm{~W} / \mathrm{m}^{\wedge} 2$ with a conversion efficiency varying from $15 \%$ to $25 \%$. While for indoor lighting scenarios, the light power intensity is around $10 \mathrm{~W} / \mathrm{m}^{\wedge} 2$ with a conversion efficiency of $2 \%$ to $10 \%$ [13].

\section{RF ENERGY}

$\mathrm{RF}$ radiation can be defined as the energy carried by electromagnetic waves with frequencies ranging from $30 \mathrm{kHz}$ to $300 \mathrm{GHz}$, illustrated in the Figure 1 . The power of an RF signal drops exponentially with unit distance from the radiation source. Therefore transmitter-receiver separation, transmission power, and antenna gain are of paramount importance for any RF energy harvesting system. Usually, when RF is used to power devices, the received power ranges between $\mu \mathrm{W}$ to a few $\mathrm{mW}$. RF energy is available 24 hours, and it can also be utilized in an indoor environment. Depending on its source, RF energy can be classified as ambient and dedicated. In ambient RF energy, the $\mathrm{SN}$ is harvesting random RF signals that are present in the environment, such as TV signals and signals from cellular base stations. While in dedicated RF energy, a transmitter transmits energy to the $\mathrm{SN}$. The network controls the dedicated RF source, and hence it has higher reliability than ambient sources. Furthermore, an RF energy harvester, unlike wind turbines, can be easily integrated into a sensor node (SN) due to the relatively limited size of the RF EH circuit [14].
Wireless power transfer (WPT) was first brought to light in 1948 when Harry Stockman published his work entitled "Communication by Means of Reflected Power" [15]. Furthermore, the first RF-powered prototype was presented by Koelle in his 1975 paper. The prototype consisted of an RF identification (RF-ID) reader with a $1 \mathrm{GHz}$ continuous wave (CW) transmitter and a $20 \mathrm{kHz}$ RF tag [16]. When the RF tag receives the $\mathrm{CW}$ signal, it rectifies the signal then sends back a $20 \mathrm{kHz}$ signal to the RF-ID reader. Furthermore, Koelle's work can be considered the first wireless powered

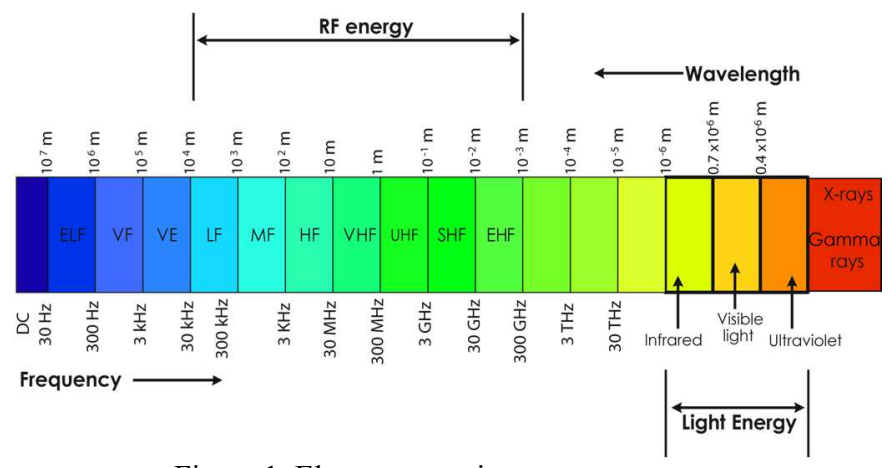

Figure 1. Electromagnetic wave spectrum.

communication system, as the RF tag rectified some of the received power and used it to transmit back to the reader.

There are two primary receiver schemes in RF energy harvesting communications: time switching (TS) and power splitting (PS). TS is a half-duplex system that requires strict synchronization. In TS, the receiver switches between two states, EH or ID. When harvesting energy, the receiver cannot decode information and vise versa. The transmission time is divided between ID and EH. As shown in Figure 2, the receiver is decoding information for a duration of $\delta$ and harvesting energy for $(1-\delta)$. The total transmission time is $\delta=1$, W is the physical bandwidth, $\eta$ is the conversion efficiency of the rectifying circuit, $h$ is the channel matrix, and $\sigma^{2}$ is the system noise. The energy-rate (E-R) ratio of TS is presented as follows [17].

$$
\mathcal{C}_{E-R}=\left\{E \leq(1-\delta) \eta h P, R \leq \delta \log _{2}\left(1+\frac{h P}{\sigma^{2}}\right)\right\}
$$

In PS, the receiver divides the power between the information decoder and the energy harvester. The signal is split according to power split ratio $\rho$ for ID and $(1-\rho)$ for energy harvesting, as shown in Figure 3 . The PS can utilize the legacy transmitters as it doesn't entail any additional components or circuity at the transmitter. The E-R region of PS can be expressed as follows [18]:

$$
\mathcal{C}_{(E-R)}=\left\{E \leq \eta \rho h P, R \leq \log _{2}\left(1+\frac{(1-\rho) h P}{(1-\rho) \sigma_{a}^{2}+\sigma_{d}^{2}}\right)\right\}
$$

In terms of hardware, An RF energy harvesting receiver should be capable of converting the received RF power to electrical power. Accordingly, three main components are required in an RF energy harvesting receiver. Antenna and matching network, charge multiplier, and energy reservoir, as shown in Figure 4. The impedance matching network prevents the received RF power from reflecting back to the antenna. 


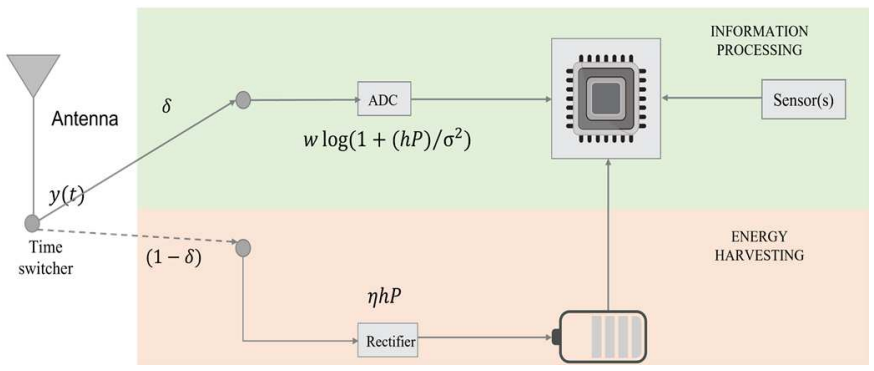

Figure 2. TS architecture.

Moreover, the matching circuit must be designed explicitly for the implemented frequency to achieve the highest efficiency.

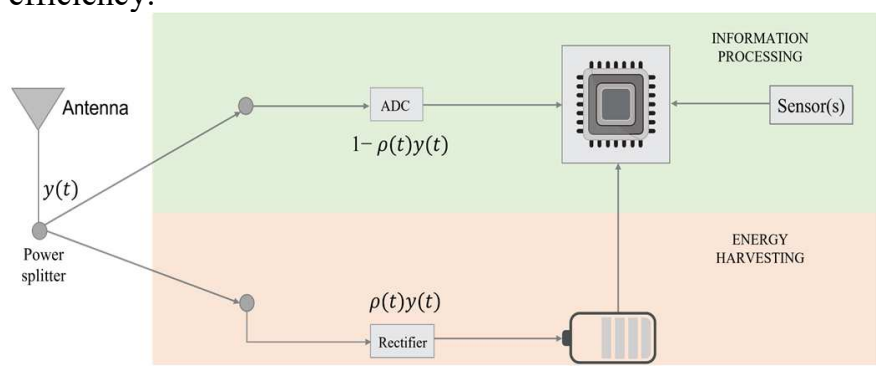

Figure 3. PS architecture.

\section{SOLAR POWERED RF POWER TRANSFER}

A solar-powered RF Transmitter represents a green energy solution in the field of wireless power transfer. In such a system, solar energy is converted into electricity using PV cells. Then the collected electricity is fed directly into an RF Oscillator or stored in an energy reservoir for future use. In some cases, where compactness is required, the solar cells are directly installed on the transmitter board or the receiver board, in the case of RFID [19]. Furthermore, in [20], a solarpowered prototype of a credit card-sized active antenna for wireless identification applications is presented.

The design of a solar-powered RF transmitter is presented in [21], Figure 5. The presented circuit collects power, up to 24 $\mathrm{mW}$, from a PV cell. Then, it uses the accumulated energy to power a Class-E oscillator, which generates and radiates electromagnetic power to low-energy sensor nodes and RFIDs.

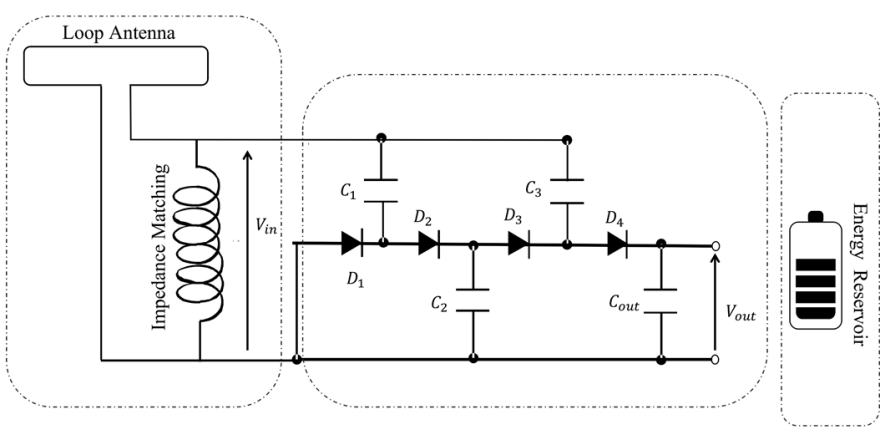

Figure 4. RF energy Harvester circuit.

In [4], a solar-powered $800 \mathrm{MHz}$ RF transmitter with an inkjet-printed antenna is presented, Figure 6 . The design in [4] is intended to be used in wireless power transfer and low-cost RFID systems.

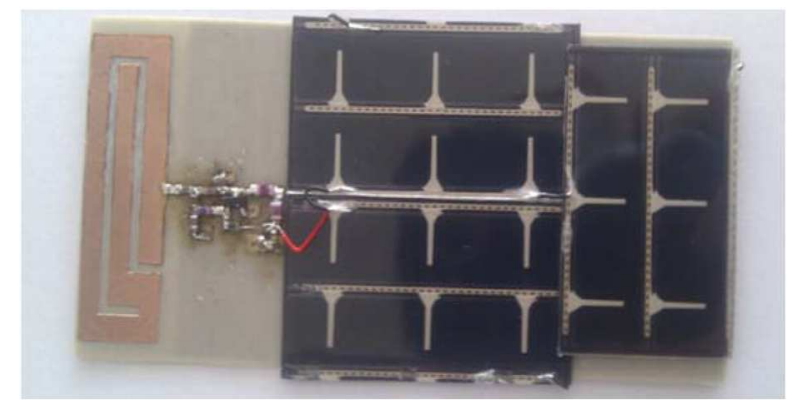

Figure 5. Prototype of a solar-powered active antenna [21].

A prototype of an early flood prediction sensor node powered by solar energy is presented in [22]. In [23], scheduling algorithms for WSN with hybrid AP powered by solar energy are studied. In [24], a wireless power sensor network is presented, Where a base station wirelessly transmits RF power to a sensor node. The authors conducted a real-life experiment using a testbed, validating that a sensor node can be charged wirelessly at a distance of $2.6 \mathrm{~m}$ from the base station, and it can stably keep the collected energy.

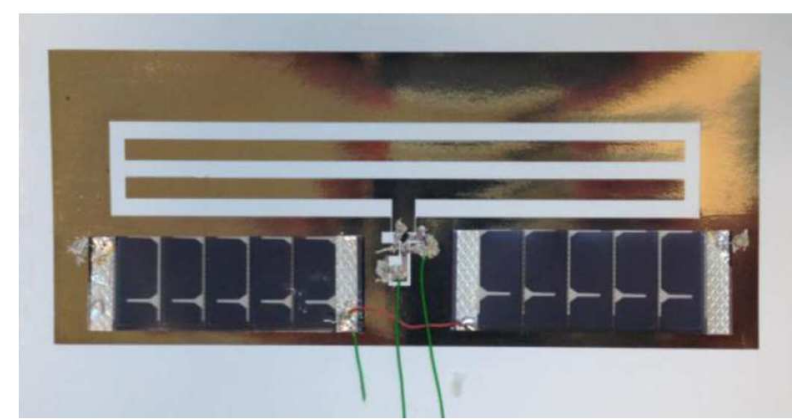
[4].

Figure 6. A solar power transmitter for wireless power transfer

In [25], the work in [24] is extended to multiple sensor nodes. A beam-splitting beamforming method that allows the base station to split the transmission power between multiple sensor nodes is studied. Accordingly, two algorithms have been presented, one for beam-splitting and the other for keeping the sensor node online. In addition, a receiver power level of $3 \mathrm{~mW}$ is achieved at the sensor node at a distance of $2 \mathrm{~m}$ from the transmitter. Furthermore, the authors in [26] studied the harvestable energy from a $2.4 \mathrm{GHz}$ WI-AP, where they deployed 23 universal software radio peripherals (USPR) inside a room. The authors concluded that a usable amount of energy could be provided to smoke detectors and thermometers through wireless power transfer.

In [27], a WSN that operates at $868 \mathrm{MHz}$ frequency is studied. The sensor can operate at a distance of $16.8 \mathrm{~m}$ when the energy transmitter power is set to $2 \mathrm{~W}$. Furthermore, the energy harvesting sensitivity at the sensor node can reach up to $-17 \mathrm{dBm}$. However, this works did not consider the high power gap between sending information and powering sensor nodes. In [28], a testbed experiment of a WSN consisting of a base station and receiving node is presented. The authors added a high-power $\mathrm{CW}$ to the transmitted signal to increase the harvestable power levels at the destination node. Accordingly, this work showed an antenna power level of 1.5 $\mathrm{mW}$ at a $3 \mathrm{~m}$ distance from the AP, achieving a harvestable power level of more than $1 \mathrm{~mW}$, Figure 7 . However, the authors did not consider the sustainability of the energy source as the high-power CW signal intended for sending energy consumes higher power than the information signal. 


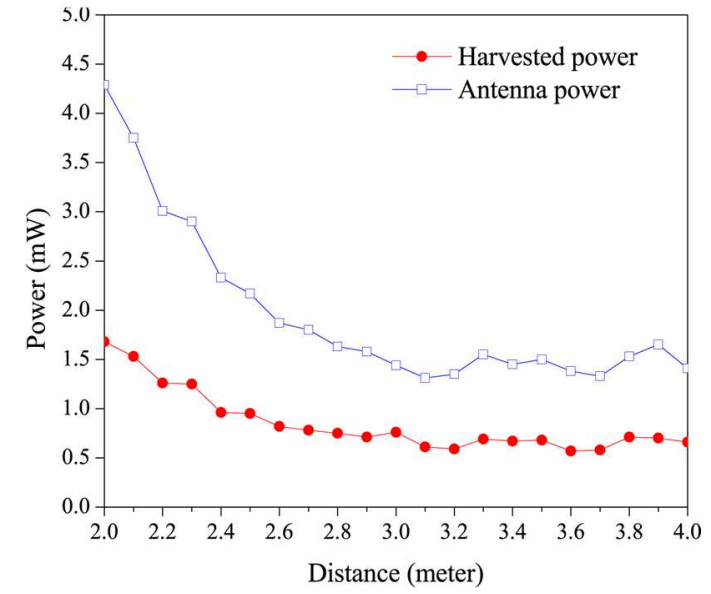

Figure 7. Sensor node antenna power and harvested power [28].

\section{RF ENERGy TRANSMITTER WITH DYNAMIC POWER ALLOCATION}

This paper presents an RF transmitter with DPA, the presented design, shown in Figure 8, consists of an RF transmitter powered by solar energy, which can be considered as an extension to our previous work in [29]. The PV array in Figure 8 converts the solar energy to electrical energy, then delivers the generated electricity to the battery. A charge controller is used to protect the battery from overchargingthe battery powers all the AP components. Moreover, the local oscillator generates a $\mathrm{CW}$ signal when biased by the battery. The output of the oscillator is split between the information processing and power transmission circuits through an RF splitter. The power circuit contains a highpower amplifier to amplify the $\mathrm{CW}$ signal. On the other hand, on the information processing side, the $\mathrm{CW}$ carrier is modulated to the information subcarriers. The information processing section contains a microcontroller (MCU) for general processing and computation, digital signal processor (DSP), which can be thought of as a special type of MCU for very high-efficiency signal processing. The digital to analog converter (DAC), as the name implies, converts the digital signal output from the DSP to an analog signal. The output from the power and information circuitries are merged through an RF combiner and transmitted through an antenna element.

In Figure 9, the power spectrum density (PSD) of TS and PS against DPA using 72 subcarriers is shown. In DPA mode,
12 subcarriers are utilized for the energy signal and the rest for the communication signal. In TS and PS all the subcarriers' power level is increased, while in DPA only the subcarriers at the carrier frequency are allocated high power, thus, decreasing the interference to outer networks. The transmitted signal is considered a DC-biased OFDM signal. The DC bias is introduced by the $\mathrm{CW}$ high power signal added to the information signal. When DC-biased OFDM is implemented, the frequency domain is limited to have selfadjoint symmetry. Hence, half of the subcarriers are utilized to transmit the communication signal to get a real-valued time-domain signal [30]. Moreover, the transmitted signal at any given time $\mathrm{t}$ is as follows:

$$
x(t)=S(t)+S_{D C}
$$

$$
S(t)=\sum_{n=1}^{N_{R}} P(t)_{n}|d(n)| \cos \left(2 \pi f_{n} t+\theta\right)
$$

Where $\mathrm{S}(\mathrm{t})$ is the transmitted communication signal, $S_{D C}$ is DC component, $P(t)_{n}$ is the power used to transmit information on the subcarrier $f_{n}$, and $d(n)$ is the real-valued communication signal.

As an example, the presented system can be considered an AP that sends energy and instructional signals to SNs and receives the sensed data from SNs. Moreover, the AP is connected to a server through a $5 \mathrm{G}$ modem for further data processing, as shown in Figure 10.

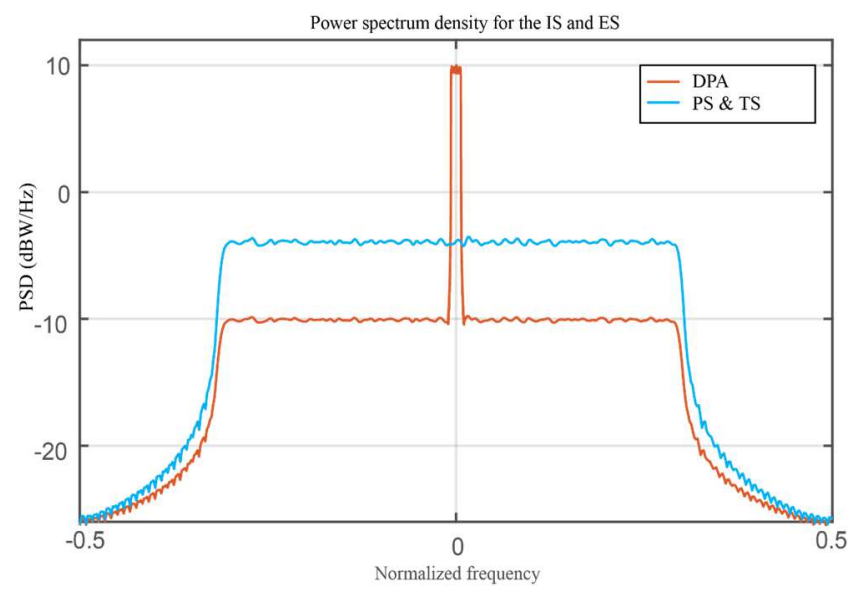

Figure 9. DPA vs. PS and TS

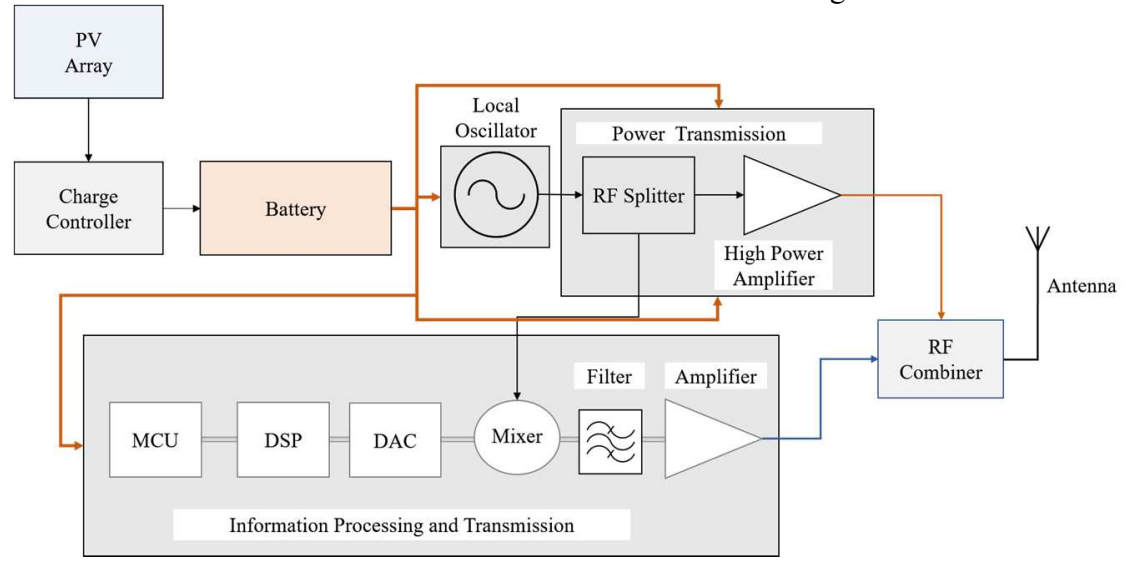

Figure 8. Solar powered Transmitter design. 
The receiver circuit consists of multiple antennas, a power combiner that combines the power from the antennas, a charge multiplier to elevate the input voltage to usable levels, and an ID section for the communication signal shown in Figure 11. The separate receiver design splits the received signal before rectification between the energy harvester and information decoder, hence increasing the power at the energy reservoir output, as shown in Figure 12.

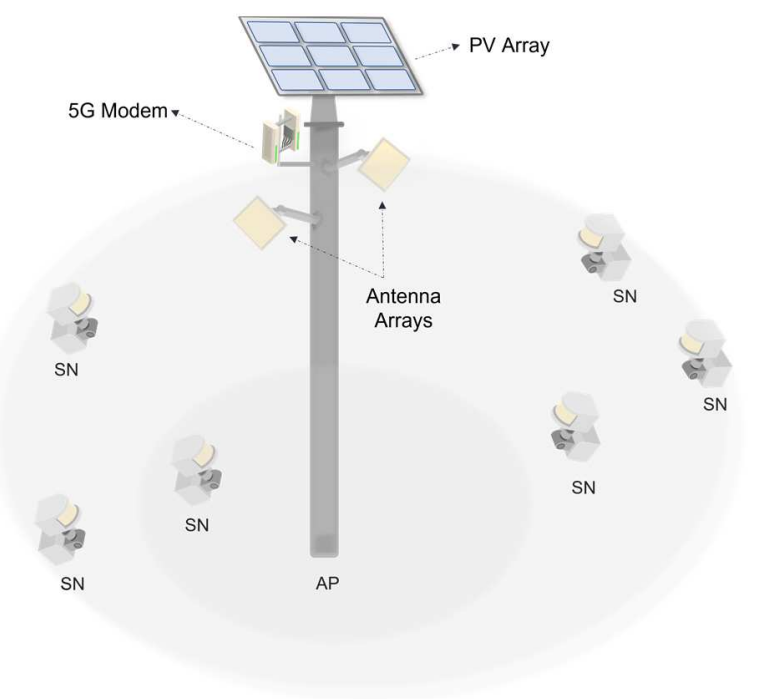

Figure 10. An example of a system design for a solar-powered RF EH WSN platform.

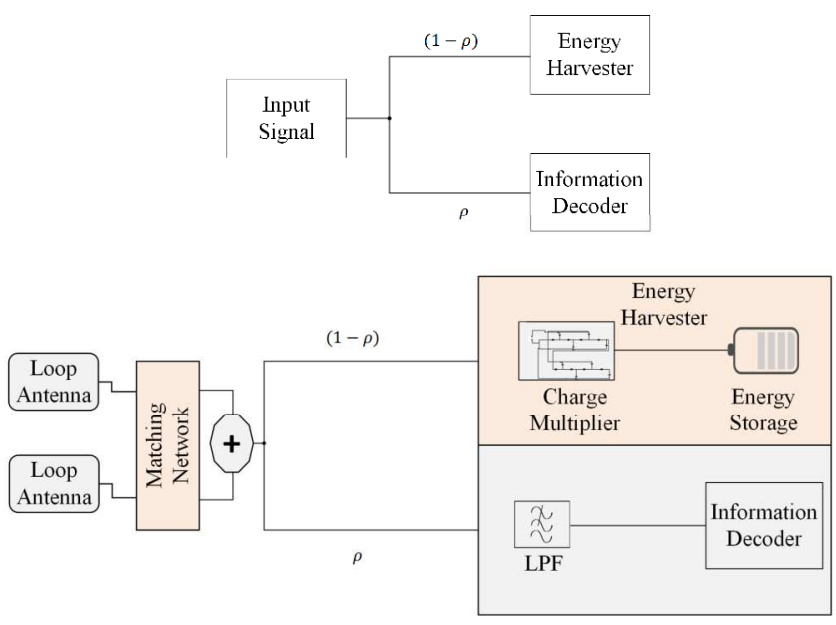

Figure 11. Sensor node receiver design[31].

\section{CONCLUSION}

Solar-powered RF energy harvesting presents a sustainable solution toward realizing green WSNs. This paper presented a solar-powered RF energy transmitter with DPA. The transmitter sends the energy signal on a high-power $\mathrm{CW}$ signal on subcarriers at the carrier frequency while the communication signal is transmitted on the remaining lowpower subcarriers. Furthermore, an $\mathrm{SN}$ receiver design with split EH and ID circuitries is adopted. The split receiver scheme increases the harvestable power level at the energy reservoir input.

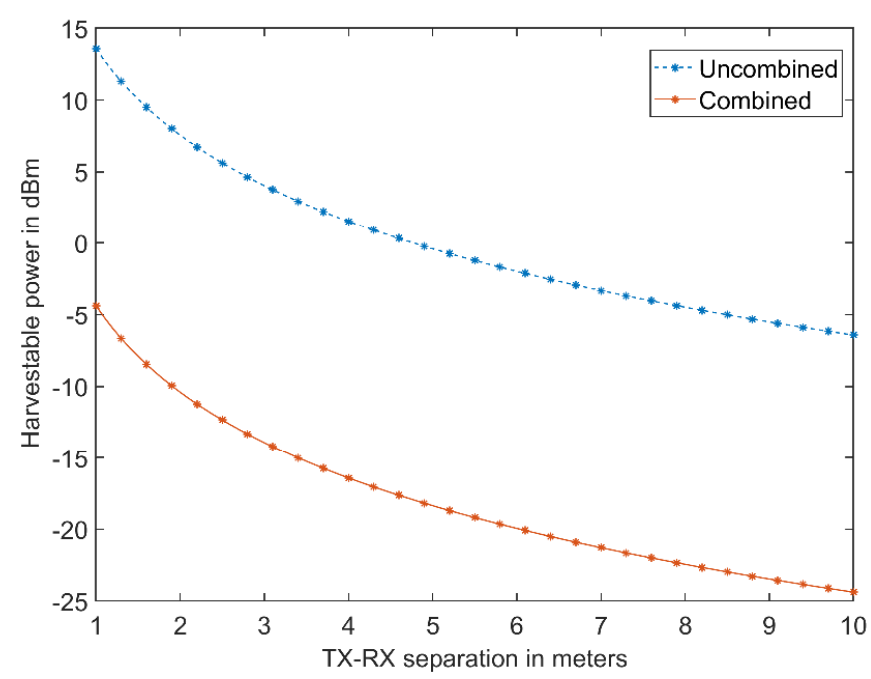

Figure 12. Combined vs. uncombined ID and EH receiver.

\section{REFERENCES}

[1] A. Viswanathan, N. B. Sai Shibu, S. N. Rao, and M. V. Ramesh, "Security Challenges in the Integration of IoT with WSN for Smart Grid Applications," 2017 IEEE Int. Conf. Comput. Intell. Comput. Res. ICCIC 2017, pp. 0-3, 2018.

[2] A. Esse, K. Abdullah, M. H. Habaebi, H. A. M. Ramli, A. L. Asnawi, and M. R. Islam, "Dynamic power allocation and scheduling for MIMO RF energy harvesting wireless sensor platforms," TELKOMNIKA (Telecommunication Comput. Electron. Control., vol. 19, no. 5, pp. 1466-1474, Oct. 2021.

S. Suman, S. Kumar, and S. De, "UAV-Assisted RFET: A Novel Framework for Sustainable WSN," IEEE Trans. Green Commun. Netw., vol. 3, no. 4, pp. 1117-1131, 2019. S. Kim, A. Georgiadis, A. Collado, and M. M. Tentzeris, "An inkjet-printed solar-powered wireless beacon on paper for identification and wireless power transmission applications," IEEE Trans. Microw. Theory Tech., vol. 60, no. 12, pp. 4178-4186, 2012.

Y. F. Su, R. R. Kotian, and N. Lu, "Energy harvesting potential of bendable concrete using polymer based piezoelectric generator," Compos. Part B Eng., vol. 153, pp. 124-129, Nov. 2018

J. Margeta and Z. Glasnovic, "Theoretical settings of photovoltaic-hydro energy system for sustainable energy production," Sol. Energy, vol. 86, no. 3, pp. 972-982, Mar. 2012.

A. E. Ahmed, K. Abdullah, M. H. Habaebi, H. A. M. Ramli, and A. L. Asnawi, "Rf energy harvesting wireless networks: Challenges, and opportunities," Indones. J. Electr. Eng. Informatics, vol. 9, no. 1, pp. 101-113, 2021.

[8] R. Ahmed, V. Sreeram, Y. Mishra, and M. D. Arif, "A review and evaluation of the state-of-the-art in PV solar power forecasting: Techniques and optimization," Renew. Sustain. Energy Rev., vol. 124, no. February, p. 109792, 2020.

[9] O. Abedinia, N. Amjady, and N. Ghadimi, "Solar energy forecasting based on hybrid neural network and improved metaheuristic algorithm," Comput. Intell., vol. 34, no. 1, pp. 241-260, 2018.

[10] J. T. Lee and D. S. Callaway, "The cost of reliability in decentralized solar power systems in sub-Saharan Africa," Nat. Energy, vol. 3, no. 11, pp. 960-968, 2018.

[11] G. K. Singh, "Solar power generation by PV (photovoltaic) technology: A review," Energy, vol. 53, pp. 1-13, 2013. 
[12] M. H. Ahmadi et al., "Solar power technology for electricity generation: A critical review," Energy Sci. Eng., vol. 6 , no. 5, pp. 340-361, 2018.

[13] C. Ó. Mathúna, T. O’Donnell, R. V. Martinez-Catala, J. Rohan, and B. O'Flynn, "Energy scavenging for long-term deployable wireless sensor networks," Talanta, vol. 75, no. 3, pp. 613-623, 2008.

[14] J. Bito, J. G. Hester, and M. M. Tentzeris, "Ambient RF Energy Harvesting from a Two-Way Talk Radio for Flexible Wearable Wireless Sensor Devices Utilizing Inkjet Printing Technologies," IEEE Trans. Microw. Theory Tech., vol. 63, no. 12, pp. 4533-4543, 2015.

[15] H. Stockman, "Communication by Means of Reflected Power," Proc. IRE, vol. 36, no. 10, pp. 1196-1204, 1948.

[16] A. R. Koelle, S. W. Depp, and R. W. FReyman, "ShortRange Radio-Telemetry for Electronic Identification, Using Modulated RF Backscatter," Proc. IEEE, vol. 63, no. 8, pp. 1260-1261, 1975.

[17] X. Zhou, R. Zhang, and C. K. Ho, "Wireless Information and Power Transfer: Architecture Design and Rate-Energy Tradeoff," IEEE Trans. Commun., vol. 61, no. 11, pp. 4754-4767, Nov. 2013.

[18] Y. Chen, Energy Harvesting Communications: Principles and Theories. wiley, 2018.

[19] F. Giuppi et al., "A $927 \mathrm{MHz}$ solar powered active antenna oscillator beacon signal generator," $R W W 2012$ - Proc. 2012 IEEE Top. Conf. Wirel. Sensors Sens. Networks, WiSNet 2012, pp. 1-4, 2012.

[20] A. Georgiadis, A. Collado, S. Kim, H. Lee, and M. M. Tentzeris, "UHF solar powered active oscillator antenna on low cost flexible substrate for wireless identification applications," IEEE MTT-S Int. Microw. Symp. Dig., pp. 79, 2012.

[21] A. Georgiadis and A. Collado, "Solar powered Class-E active antenna oscillator for wireless power transmission," IEEE Radio Wirel. Symp. RWS, pp. 40-42, 2013.

[22] P. Visconti, P. Primiceri, and C. Orlando, "Solar powered wireless monitoring system of environmental conditions forearly flood prediction or optimized irrigation in agriculture," ARPN J. Eng. Appl. Sci., vol. 11, no. 7, pp. 4623-4632, 2016.

[23] C. Li, K.-W. Chin, and C. Yang, "Complete Target Coverage in Radio Frequency and Solar-Powered Sensor Networks," IEEE Syst. J., pp. 1-11, 2020.

[24] K. W. Choi, L. Ginting, P. A. Rosyady, A. A. Aziz, and D. I. Kim, "Wireless-Powered Sensor Networks: How to Realize," IEEE Trans. Wirel. Commun., vol. 16, no. 1, pp. 221-234, 2017.

[25] K. W. Choi, P. A. Rosyady, L. Ginting, A. A. Aziz, D. Setiawan, and D. I. Kim, "Theory and Experiment for Wireless-Powered Sensor Networks: How to Keep Sensors Alive," IEEE Trans. Wirel. Commun., vol. 17, no. 1, pp. 430-444, 2018.

[26] O. Georgiou, K. Mimis, D. Halls, W. H. Thompson, and D. Gibbins, "How Many Wi-Fi APs Does it Take to Light a Lightbulb ?," IEEE Access, vol. 4, pp. 3732-3746, 2016.

[27] M. Pizzotti et al., "A long-distance RF-powered sensor node with adaptive power management for IoT applications," Sensors (Switzerland), vol. 17, no. 8, 2017.

[28] K. W. Choi et al., "Simultaneous Wireless Information and Power Transfer (SWIPT) for Internet of Things: Novel Receiver Design and Experimental Validation," IEEE Internet Things J., vol. 7, no. 4, pp. 2996-3012, 2020.

[29] A. Esse, K. Abdullah, M. H. Habaebi, and H. A. M. Ramli, "Dynamic Power Splitting Simultaneous Wireless Information and Power Transfer Split Receiver for Wireless Sensor Networks," IEEE Access, vol. 9, pp. 129407-129416, 2021.

[30] A. A. Sharifi, "A new post-coding approach for PAPR reduction in DC-biased optical OFDM systems," Optoelectron. Lett., vol. 15, no. 4, pp. 302-305, 2019.

[31] A. E. Ahmed, K. Abdullah, and M. H. Habaebi, "Wavelet Modulation For Energy Efficient Wireless Sensors Networks with Simultaneous Wireless Information and Power Transfer," pp. 308-313, Jul. 2021. 